\title{
SIMULATION OF TRAFFIC FLOWS ON ROAD SEGMENTS USING CELLULAR AUTOMATA THEORY AND QUASIGASDYNAMIC APPROACH
}

\author{
N.G. CHURBANOVA ${ }^{1,2^{*}}$, A.A. CHECHINA ${ }^{1}$, M.A. TRAPEZNIKOVA ${ }^{1,2}$, \\ P.A. SOKOLOV \\ ${ }^{1}$ Keldysh Institute of Applied Mathematics RAS, Moscow, Russia \\ ${ }^{2}$ Moscow Automobile and Road Construction State Technical University (MADI), Moscow, Russia \\ * Corresponding author. E-mail: nataimamod@mail.ru
}

DOI: 10.20948/mathmontis-2019-46-7

Summary. The research deals with the creation of mathematical tools for the simulation of vehicular traffic flows on complex urban transport networks using modern supercomputers. The goal of the present paper is further development of micro- and macroscopic models created by the authors earlier. The proposed $2 \mathrm{D}$ microscopic model is based on the cellular automata theory. In this work algorithms taking into account various driving strategies have been incorporated into the model. The model is implemented as a program package that includes User interface and Visualization module. The macroscopic model uses the continuous medium approximation: it is constructed by analogy with the quasigasdynamic system of equations. The one dimensional version is proposed in the paper, nevertheless, it allows reproducing changes in the number of lanes as well as possible road entrances and exits. Parallel algorithms adapted to high-performance computing systems have been created for both models, ensuring rapid computations on city road networks.

\section{INTRODUCTION}

Mathematical modeling of traffic flows is a topic of increasing interest for lots of scientists all over the world. Due to the permanent growth of the number of vehicles, modern cities require solutions to support traffic management, both short-term and long-term, and the rising capacity of modern supercomputer systems allows using more detailed and precise models for computations. At the same time, for different types of models different tasks are to be fulfilled. The microscopic models (these include cellular automata models) consider various aspects of real life driver behaviour, resulting in more fluctuations inside the flow that consequently leads to reproducing experimentally observed features of traffic. The macroscopic (gasdynamic) models are means for providing a general picture for traffic situation on a network while avoiding excessive computational cost.

The first approach under consideration is the microscopic approach. As in atomic models describing the interaction of atoms and molecules [1-5], here we consider the interaction of individual particles represented in the form of cars with their drivers. Cellular automata (CA) models are a special type of microscopic models. The CA theory, first proposed by John von Neumann in the mid-twentieth century, has got applications in many fields of science. It helps to predict economic, social, technical, biological and other processes. The first application of the theory of cellular automata to the traffic flow simulation was proposed in [6]. But

2010 Mathematics Subject Classification: 93C52, 68W10, 68W05, 05C21.

Key words and Phrases: Mathematical modeling, Traffic flows, Cellular automata, Parallel computing. 
especially rapidly this trend began to develop since 1992, when Kai Nagel and Michael Schreckenberg applied the CA theory to transport modeling [7]. Scientists from around the world have created many variants of traffic flow models based on it. One of the most interesting reviews on cellular automata models is given in [8]. It is noted that the main goal of CA models is to describe the macroscopic characteristics of traffic flows using a simple description of microscopic interactions.

One can trace the further development of this direction, for example, in the following works. Article [9] suggests stochastic version of the CA model. Article [10] discusses the simulation of dynamic systems on toroidal structures. In $[11,12]$ on the basis of the CA model investigations are carried out to illustrate the three-phase Kerner theory [13]. Article [14] is devoted to the same issues. In [15] the effect of the difference in driver psychology on flow stability on a two-lane highway is estimated using the CA model. The article also shows the influence of safety parameters on the volume of traffic. Some works [16-21] deal with the adaptation of the CA model to various real transport situations, such as the impact of the distribution of lanes and turn signals at the intersection on the total traffic capacity, accounting for interaction with neighboring vehicles, accounting for pedestrian crossings, traffic on a U-shaped turn, the interaction of cars with buses and bicycles, addresses traffic safety issues. Some of these transport situations are considered by the authors in the present paper.

Previously, the CA approach seemed to be the most promising for a detailed description of local road situations at short distances, since the models are quite flexible due to the ability to implement any driver strategy without significant algorithmic costs. However, the capacity of modern ultra-high-performance computing equipment allows models of this type to be successfully used to simulate traffic on large road networks.

The second approach discussed in this paper uses the continuous medium approximation. An approach of this kind is widely used in different branches of science, such as gas dynamics [22-25], flows in porous media [26] and many others. To clarify the basic regularities of dense traffic flow, it is convenient to use the continuous medium approximation in traffic simulations, i.e. to use macroscopic models (see [27]). In contrast to microscopic models, the main objects of study in such models are fields of the average vehicle speed and the density of vehicular flow. Recently, the number of works devoted to macroscopic models has slightly decreased, however, there are some cases when the macroscopic description is more computationally advantageous and gives more clear representation of the average characteristics of the traffic flow.

A significant amount of works is devoted to models such as Lattice Boltzmann hydrodynamic models. The following articles can be listed here. A theoretical analysis of these types of models is presented in [28]. In [29] such characteristics of drivers as "timidity" or "aggression" are taken into account. In $[30,31]$ the influence of the human factor on the stability of a two-way flow is investigated. The authors of article [32] propose a new lattice model, which helps to study how the driver's memory volume affects the dynamics of the traffic flow. Analysis of the linear stability demonstrates that the temporary length of the driver's memory has an important effect on the stability of the traffic flow. And in article [33] the same authors propose an extended one-dimensional hydrodynamic lattice model to study flow dynamics when driving along a curved road. They obtain the stability condition via the linear stability analysis. The result of the analysis indicates that the radian of a curved road plays an important role in influencing the flow stability. In addition, compared with other 
segments of the curved road, the flow easily becomes unstable at the road entrance and exit. Article [34] also explores movement along a curved road. On the basis of the lattice model it is confirmed that a curved road negatively affects the stability of the traffic flow.

As follows from the above review, traffic flow simulation has been actively developed in previous decades. Nevertheless an adequate description of the interaction of traffic flows in the network nodes under conditions of congested traffic requires further modernization of existing models and methods for their computer implementation. In view of the significantly increased loading of city transport networks in recent years, a separate independent task is to create software for the efficient use of parallel computer technologies.

The aim of the present paper is the development of mathematical tools for modeling the dynamics of traffic flows on complex fragments of road networks that are structural elements of a branched transport system of a metropolis. For this purpose the paper discusses two possible ways of solving the given problem. Previously the authors created the original microscopic model based on the cellular automata theory and the macroscopic model using the ideology of the quasigasdynamic system of equations. In the current research these models undergo the needed modification.

In the field of micromodeling, the goal is the extension of the CA model by introducing new algorithms that describe a variety of traffic situations, as well as possible driver strategies. Improved algorithms of lane changing, crossroad overcoming, queue forming, moving on the road with complex geometry, driving around obstacles are to be created. Algorithms depicting different driving strategies are also to be included. Another goal is to create a so-called "slow-to-start" model for reproducing real fluctuations that occur in congested traffic.

In the field of macromodeling the main goal is the development of the gasdynamic type model with a simple enough structure but with possibility to describe the movement over crossroads, roads with the changing number of lanes and to account for the existence of onramps and off-ramps. In paper [35] a 2D macroscopic model was proposed by analogy with the quasigasdynamic (QGD) system of equations designed to predict a wide class of compressible gas flows including low Mach number flows (see [36]). However, when modeling traffic at complex junctions and interchanges, and especially in numerical implementation, such a description may cause additional difficulties. At the same time, in many practically important cases a one-dimensional description can be sufficient to study peculiarities of vehicular flow and to obtain qualitatively correct results.

A common task for models of both types is their adaptation to distributed memory multiprocessor systems to make the calculations most efficient.

The approaches are to be verified by various numerical experiments, including test predictions of traffic on signalized intersections at different traffic light regimes.

\section{THE ORIGINAL 2D CA MODEL}

The original cellular automata model created by the authors presents a generalization of the classic Nagel-Schreckenberg model [7] to the multilane case with various driver behaviour algorithms included.

The road is divided into equal cells. As is usual for traffic CA models, a cell is 7.5 meters long and one lane wide, the time step is 1 second. The cell can be either empty or occupied by a single vehicle (Fig. $\left.{ }^{\circ} 1\right)$. 


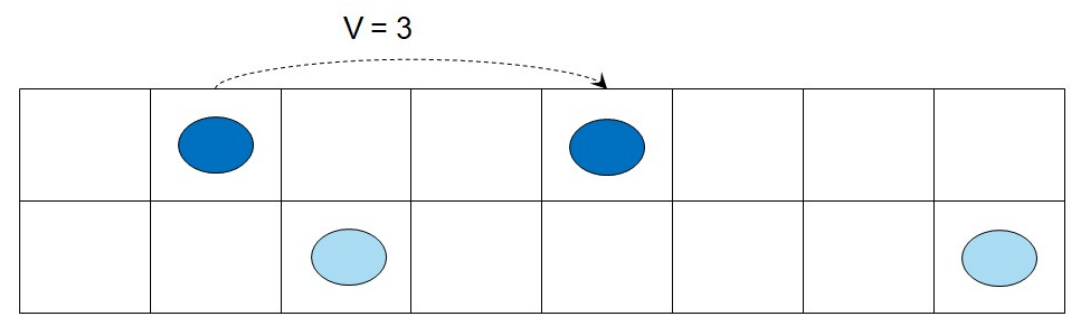

Figure 1. The computational domain for CA-model

Each car has a set of parameters: unique ID, maximum speed $-V_{\max }$, current location (the road number, the lane number and the cell number), current speed - from 0 to $V_{\max }$ and final destination; its driver can be 'cautious' or 'aggressive', 'cooperative' or not. These parameters, especially location and speed can change at each time step, it depends on the situation around the car.

Each time step cell state update is carried out according to the following rules:

- a vehicle changes a lane if it is necessary (to reach the desired destination or to drive around an obstacle), it is advantageous for a driver (leads to speed increase and/or density decrease) and it is possible (i.e. if the lane change is allowed and the target cell is empty);

- a vehicle moves along the road according to the classic rules for the one-lane traffic [7].

These rules consist of the substeps represented as a block-scheme (Fig. 2) for each car, $d-$ the distance between the current car and the next one in front of it.

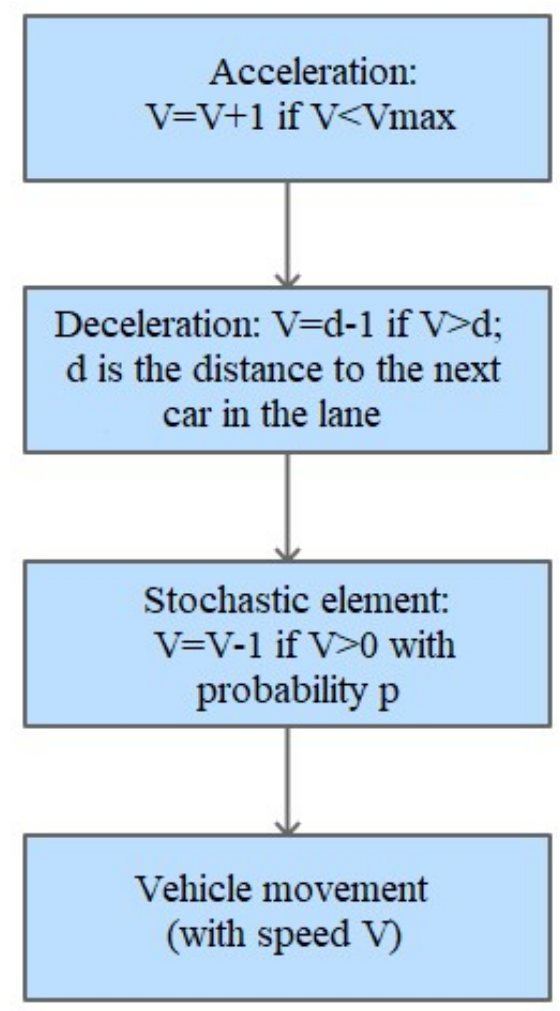

Figure 2. Block-scheme for the 1D Nagel-Schreckenberg model 
The first step reflects the general desire of all drivers to drive as quickly as possible. The second guarantees no collisions with vehicles in front. An element of stochastics, taking into account randomness in the behaviour of drivers, is introduced in the third step. On the fourth, the state is finally updated for this movement participant.

\section{ALGORITHMS INCLUDED IN THE CA MODEL}

\subsection{Changing lanes}

The rules of changing lanes can depend on various conditions, i.e. road segment, road signs, driver behaviour.

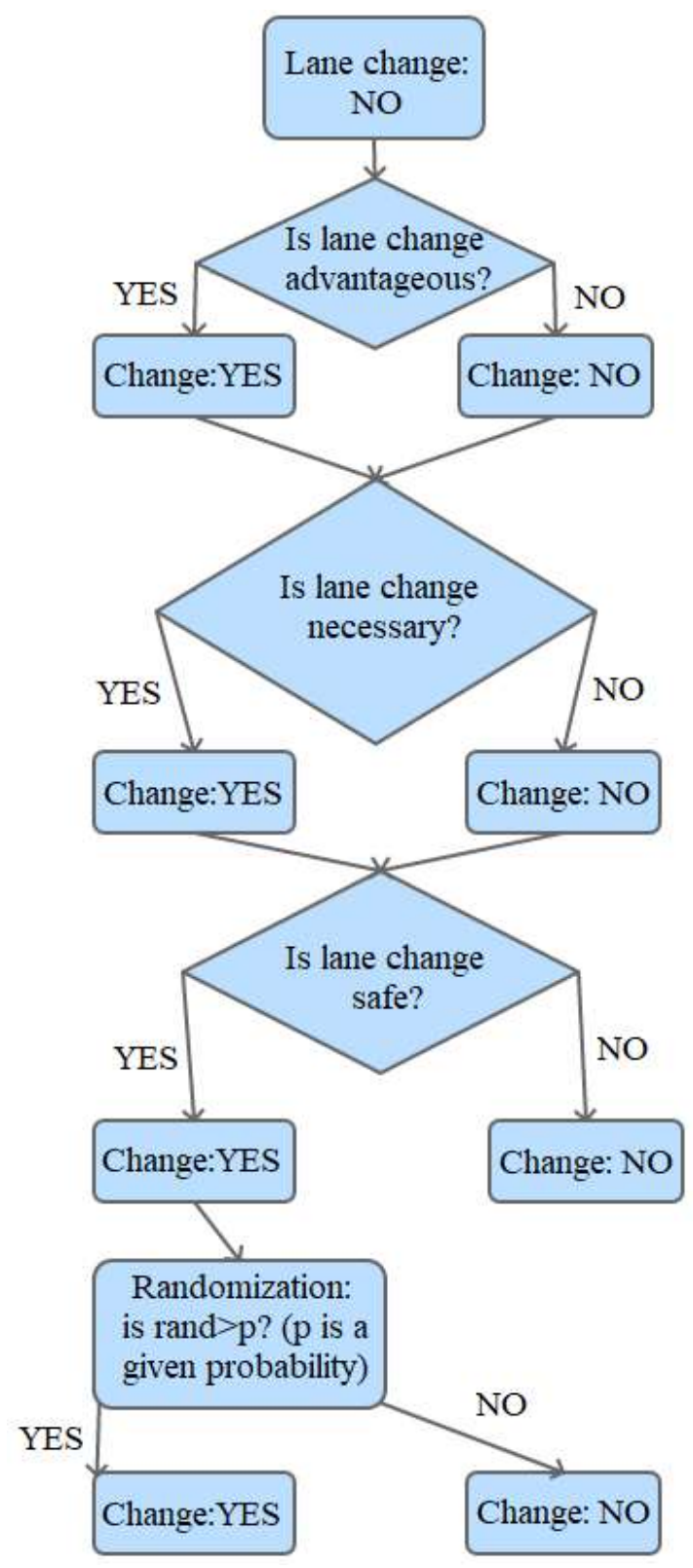

Figure 3. Block-scheme for the lane change algorithm 
The block-scheme for the basic algorithm of lane changing is presented in the Fig. ${ }^{\circ} 3$ : Lane changing takes place under following conditions:

- a vehicle is located in a domain where lane change is allowed;

- lane change leads to the speed increase (density decrease) or is necessary to reach the destination;

- there is an obstacle not far away on the current lane;

- the target cell is empty;

- the safety condition is satisfied - on the target lane the distance behind is greater or equal to the maximal/current velocity of the previous car (cautious/aggressive drivers), the distance in front is greater or equal to the concerned vehicle velocity;

- lane change takes place with a given probability.

The described algorithm is basic and its variations are included as an integral part in all subsequent algorithms for the movement of cars on various elements of the transport network. The algorithms described below have a much more complex structure, so their graphical representation in the form of block-schemes is rather bulky for the article format.

\subsection{The algorithm of crossroad overcoming}

- Within 100 meters before traffic lights the vehicle changes lane under its purpose according to the road laws.

- A vehicle accelerates or decelerates according to the Nagel-Schreckenberg model.

- Additional speed decreasing takes place under the following conditions:

- if a vehicle is located near the turning point (at the turning point it stops);

- if a traffic light is red;

- if there is the collision threat on the crossroad.

- A vehicle moves under the foregoing rules with randomization.

- A vehicle turns if it is located in the turning point and has got the corresponding target.

\subsection{Forming the entrance queue}

According to the entrance flow value for the given direction that is set by user before the calculation, time steps on which new vehicles should be added in the system are specified. At a given time step the vehicle appears in the entrance cell of the right lane if it is not occupied by another vehicle. If it happens to be occupied, other lanes are checked. If there is a traffic jam and all the entrance cells in the given direction contain vehicles already, the car can't enter and it is added into the queue. As soon as one of the entrance cells vacates, the car appears on the road.

\subsection{Road narrowing/widening}

Each driver is able to "see" if the road narrows down some distance ahead. In this case, the driver tries to change lane if his lane is going to disappear, even if the speed in the target lane is lower than the speed in his current lane and the traffic over there is denser. Some drivers in the target lane, if they are "cooperative", can stop to let the driver change his lane.

In case of the road widening, drivers react only when they reach it, following the general "lesser density/higher velocity" rule, i.e., seeing the empty lane, they move there for more comfortable driving. 


\subsection{Driving around an obstacle}

If there is an obstacle in the middle of the road (for example, a broken car or cars after a road accident), a driver is able to "see" it before it reaches it, like in the case of a road narrowing. He also tries to drive around it, first determining which direction of lane changing is preferable, and then he changes lanes till he reaches a free one, with or without help of "cooperative" drivers.

\subsection{Different driving strategies}

Each driver has his own driving style: ones are more determined, change lanes more easily, others are more cautious, they wait for a larger gap to move to the neighbouring lane. Their behaviour influences the overall situation on the road. To reflect that, the following driver types were included in the model:

- "Cautious" drivers change lanes only if the gap between the target cell and the first occupied cell upstream is larger than the maximum speed;

- "Aggressive" drivers change lanes if the gap between the target cell and the first occupied cell upstream is larger than the actual speed of the vehicle which is situated in the first occupied cell;

- "Cooperative" drivers can be either cautious or aggressive. The percentage of cooperative drivers in the system can vary. These drivers:

- slow down $(\mathrm{V}=\mathrm{V}-1 ; \mathrm{V} \geq 1)$, if they see a traffic jam before an obstacle or a road bottleneck on the neighbouring lane;

- if there is a jam before the obstacle on the neighbouring lane, and there are drivers that want to change their lane, cooperative drivers stop and let them pass;

- wait for several time steps if the car from the other lane can't move immediately, because the target cell is occupied by another car.

\section{THE 'SLOW-TO-START' VERSION OF THE CA MODEL}

According to the classic one-lane Nagel-Schreckenberg rules, the driver checks if the next cell is empty, and if it is, he starts moving. But there is another class of models - 'slow-tostart' - where vehicles begin their movement only on condition that there is more than one free cell in front of them (see [8]). This rule was included in the model so that cars did not disperse too quickly from the place of the traffic jam. It allows to reproduce the effect of hysteresis that is observed during the transition from the free flow phase to the synchronized flow phase, depending on random processes in the traffic flow.

According to the three phase theory by B. Kerner [13], there are three phases in the traffic flow: $\mathrm{F}$ is the phase of free flow, $\mathrm{S}$ is the phase of synchronized flow and $\mathrm{J}$ is the phase of wide moving jams. Due to the instability of the flow, for example, due to the effect of overacceleration (see [7]), phase transitions can occur spontaneously. As experimental data show, models of the 'slow-to-start' class reproduce such phase transitions more successfully.

To satisfy the 'slow-to-start' rule, a set of appropriate conditions was added to the created model. The results obtained via this model can be found in paper [37]. 


\section{NUMERICAL REALIZATION}

The created program package consists of two modules: the Computational module that carries out calculations and the User Interface and Visualization module that serves the purpose of getting the initial data from a user, transferring it to the Computational module, getting results back and providing their visual interpretation.

The code is written in $\mathrm{C} / \mathrm{C}++$ and uses the MPI library for parallel calculations. Separate sub-programmes simulate traffic on different types of road elements in parallel, with data exchange on the boundaries.

The results of computations for some standard road elements are shown in Fig. 4. Different colours of cars represent different destinations attributed to them. Fig. 4(a) and Fig. 4(c) represent signalized intersections (T-cross and X cross respectively). In Fig. 4(b) an U-turn on a road with a wide median is shown. In Fig. 4(d), where the road with an accident is presented, the black circle represents an unmoving car that experienced the accident. In Fig. 4(e) an on-ramp is shown.


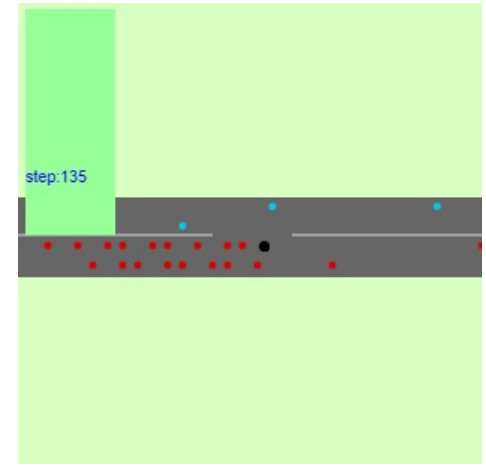

$4(d)$

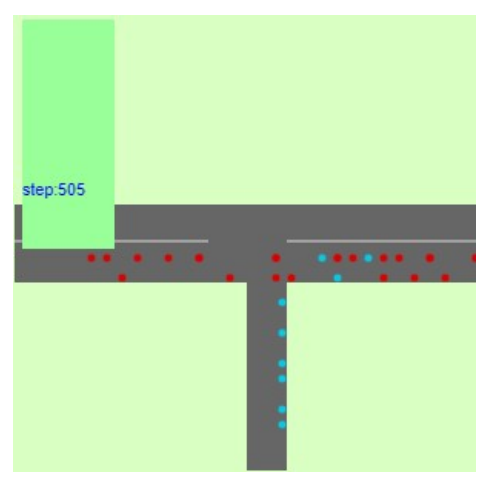

$4(e)$

Figure 4. Basic road fragments

At each time step processors exchange information whether any vehicles are crossing the boundaries during this step. If the answer is positive, the data regarding those vehicles is packed and sent/received, and the cars appear on the next road fragment. In order to avoid the situation where the target cell is already occupied, the information about all vehicles that stopped near the beginning of the road fragment is collected. If there is a traffic jam on the road, the drivers from the previous (upstream) fragment that are nearing its end can "see" it 
and slow down or stop if necessary.

\section{TEST PREDICTIONS FOR THE CA MODEL}

The models have been verified by various numerical experiments (see, for example, $[38,39])$, including the comparison with experimental data [37].

For further comparison and verification of the created models a few test problems were researched. The first one is modelling traffic on a small road network of two neighbouring intersections (see Fig. 5). It consists of two elements: an U-turn on the road with a median wide enough to fit in a car, and a signalized X-crossroad, that are connected by data exchange on the boundaries via MPI. Once the car leaves its element, it appears on the next, obtaining a new destination (since its previous destination is considered to be reached).



Figure 5. Simulating traffic on two neighbouring intersections: U-Turn $+X$-Crossroad

Another test problem is modeling traffic on a small network which consists of two signalized intersections (Fig. 6). When a car approaches the boundary with another element, the possibility of entering is checked. If the desired cells are occupied by other cars waiting for the green light on the target element, the car stops and waits until one of these cells is empty.

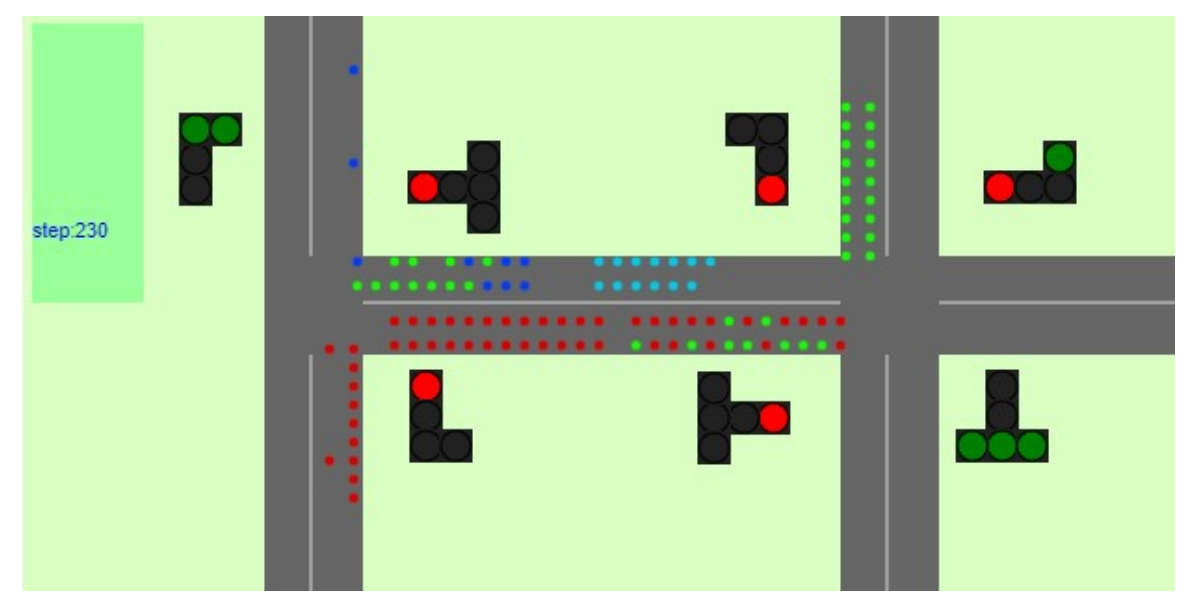

Figure 6. Simulating traffic on two neighbouring intersections: T-Crossroad + X-Crossroad 
Along with the visual representation of what is happening with traffic on this part of the network, various average characteristics can be obtained [37].

\section{THE QGD APPROACH TO TRAFFIC FLOW MODELING}

\subsection{The governing macroscopic model}

In case of congested traffic when average quantities of traffic flow are of primary interest the continuous medium approximation is convenient to employ, i.e. to use macroscopic models. In contrast to microscopic models, the main objects of study in such models are fields of the average vehicle speed and the density of vehicular flow. The most popular models of this type are models like gas-dynamic ones.

For many practically important problems a one-dimensional description is sufficient to study peculiarities of vehicular flow and to obtain qualitatively correct results. The paper focuses on a $1 \mathrm{D}$ variant of the quasigasdynamic equations system for vehicular traffic flow. It can be written as follows:

$$
\begin{gathered}
\frac{\partial \rho}{\partial t}+\frac{\partial \rho V}{\partial x}=\frac{\partial}{\partial x} \frac{\tau}{2} \frac{\partial\left(\rho V^{2}+P\right)}{\partial x}+F_{\rho} \\
\frac{\partial \rho V}{\partial t}+\frac{\partial \rho V^{2}}{\partial x}=f-\operatorname{grad} P+\frac{\partial}{\partial x} \frac{\tau}{2} \frac{\partial\left(\rho V^{3}+P V\right)}{\partial x}+F_{V}
\end{gathered}
$$

Here $\rho\left[\frac{\mathrm{veh}}{\mathrm{km} \cdot \text { lane }}\right]$ is the traffic density, $V\left[\frac{\mathrm{km}}{\mathrm{h}}\right]$ is the spatial average speed of vehicles.

The above system belongs to macroscopic models of the second order [27]. It includes two equations in the form of conservation laws for obtaining the density (1) and the speed (2). Since the solution of the system can be occasionally discontinuous, i.e. so-called traffic shock-waves can take place, as a rule diffusion terms are introduced into the equations for solution smoothing. The QGD system possesses such a property initially. One of the basic assumptions for the QGD system is existence of additional mass flux that ensures a smooth solution at the reference scale of the medium (see [36]). The right-hand sides of equations (1) and (2) include such fluxes. By analogy with gas dynamics minimal reference time and space scales are introduced for traffic flows in order to satisfy the continuous medium approximation. The small parameter $\tau$ is interpreted as a reference time that means the time interval in which several vehicles cross a given point of the road. As a reference length the distance between vehicles for the given speed can be considered.

Equations (1)-(2) employ the listed below additional functions related specifically to transport problems.

The analogue of pressure: $P(\rho)=\frac{\alpha \rho^{\beta}}{\beta}$.

Phenomenological constants are taken from works by other authors as $\alpha=60 \frac{\mathrm{km}^{2}}{\mathrm{~h}^{2}}, \beta=2$. 
The accelerating/decelerating force: $f=a \rho$. Here $a$ is the relaxation term reflecting adaptation of the speed to the equilibrium speed: $a=\frac{V_{e q}(\rho)-V}{T}$.

The involved equilibrium speed $V_{e q}(\rho)$ has the sense of the optimal speed under the given conditions. It is a function depending only on the density and obtaining from the fundamental diagram. In the current investigation the parabolic fundamental diagram is used:

$$
Q_{e q}(\rho)=\rho V_{0}\left(1-\frac{\rho}{\rho_{j a m}}\right) \text {. }
$$

Taking into account the relation $Q_{e q}=\rho V_{e q}$ we get the next dependence of the equilibrium speed on the density:

$$
V_{e q}(\rho)=V_{0}\left(1-\frac{\rho}{\rho_{\text {jam }}}\right)
$$

Here $V_{0}$ is the free traffic speed, $\rho_{j a m}$ is the density at which vehicles stop moving ("traffic jam"). In computations the next values are used: $V_{0}=90 \frac{\mathrm{km}}{\mathrm{h}}, \rho_{j a m}=120 \frac{\mathrm{veh}}{\mathrm{km} \cdot \text { lane }}$.

The function $T$ depends on the density:

$$
T(\rho)=t_{0}\left(1+\frac{r \rho}{\rho_{\text {jam }}-r \rho}\right) \text {, where } t_{0}=50 \text { and } r=0.95 \text { are the model parameters. }
$$

The source functions in the right-hand sides of equations (1)-(2) are equal to zero if the road is homogeneous, that is the number of lanes does not change and there are no entrances/exits. If there is a change in the number of lanes, then the concept of the real function $I$, which has the meaning of the number of lanes, is introduced [27]. It does not change abruptly, but is piecewise linear or may have a more complex form. When the number of lanes changes, it takes not integer (i.e. real) values on a certain distance before the narrowing/widening. The integer part of this value reflects the contribution of the continuing lanes, and the mantissa is the contribution of the closing/opening lane to the traffic movement as a whole.

In addition, if there are entries/exits, the corresponding source terms appear in the righthand sides of the equations. If by analogy with [27] we introduce the concept of "the effective source density", denoting it $v_{r m p}$, the right-hand side of equation (1) takes the following form:

$$
F_{\rho}(x, t)=-\frac{\rho V}{I} \frac{\mathrm{d} I}{\mathrm{~d} x}+v_{r m p}(x)
$$

where 


$$
v_{r m p}(x, t)=\left\{\begin{array}{l}
\frac{Q_{r m p}(t)}{I L_{r m p}} \text { if } x \text { is inside the entry / exit zone, } \\
0 \quad \text { otherwise. }
\end{array}\right.
$$

Here $Q_{r m p}$ denotes the incoming (from the entry) or outgoing (from the exit) traffic flow, $L_{r m p}$ is the acceleration band length - the length of the on-ramp or off-ramp. The source term for the momentum equation (2) can be also obtained after [27]. For the conservative form of the equation it will be as follows:

$$
F_{V}(x, t)=-\frac{\rho V^{2}}{I} \frac{d I}{d x}+V v_{r m p}(x)+\rho A_{r m p}
$$

where

$$
A_{r m p}=\frac{\left(V_{r m p}-V\right)\left|Q_{r m p}\right|}{\rho I L_{r m p}},
$$

$V_{r m p}$ is the speed of on-ramp vehicles merging or diverging the main road, $V_{r m p}<V$.

Differential equations should be supplemented at boundary points of the computational domain by boundary conditions of the first or second kind for the density and velocity that depend on the specific formulation of the problem.

\subsection{Numerical implementation}

For numerical implementation of system (1)-(2) an explicit finite-difference method is used. The next two-level scheme is applied, convective terms are approximated by central differences [40]:

$$
\begin{gathered}
\frac{\hat{\rho}-\rho}{\Delta t}+(\rho V)_{x}^{0}=0.5 \tau\left(\rho V^{2}+P\right)_{\overline{x x}}+F_{\rho}, \\
\frac{\hat{\rho}-\rho}{\Delta t}+\left(\rho V^{2}+P\right)_{x}^{0}=f+0.5 \tau\left(\rho V^{3}+P V\right)_{\bar{x} x}+F_{V} .
\end{gathered}
$$

The conditional stability of schemes is ensured by the presence of diffusion terms in the right hand sides. As the numerical implementation is based on explicit schemes its parallelization can be performed with high efficiency. The parallel algorithm is focused on the use of distributed memory supercomputers.

For the interaction of parallel processes in the code the MPI (Message Passing Interface) technology is employed. Parallelization is based on the principle of geometrical parallelism. For macroscopic traffic flow computations the algorithm with partitioning of the computational domain (the road network) into subdomains was proposed. Each subdomain represents one segment of the network connecting two neighbouring nodes (intersections). In network nodes the data exchange between adjacent segments occurs. Splitting is carried out in such a way that two neighbouring sections have two common points for the correct implementation of boundary conditions at bordering points of subdomains. Thus, each section 
of the road is calculated on a separate processor, transmitting and synchronizing data via MPI operations.

Further calculations were carried out at Keldysh Institute of Applied Mathematics RAS on the MVS-Express supercomputer, which has a distributed memory architecture (see [32]).

\section{TEST PREDICTIONS FOR THE QGD TRAFFIC MODEL}

As in case of the CA model the QGD traffic model verification was performed by test problems on signalized intersections. In test predictions the intersection is represented as a graph, at parallelization each subdomain corresponds to the graph edge.

In the signalized intersection test problem a traffic light is placed in the graph node, allowing one or another entrance flow alternately pass through the intersection in accordance with the traffic light phase.

For the flow for which the green light is on, conditions of matching are set on the boundary between neighboring subdomains - a simple exchange of the boundary values of densities and velocities takes place. For the flow for which the red light is on, the density and the velocity at the point of the traffic light location are discontinuous: on the left of the traffic light the velocity equals zero, the density increases up to the maximum. On the right of the traffic light the movement continues. Equations (1)-(2) in this point are simplified to the next ones (the acceleration and the pressure gradient become equal to zero):

$$
\begin{gathered}
\frac{\partial \rho}{\partial t}=-\frac{\partial \rho V}{\partial x}, \\
\frac{\partial \rho V}{\partial t}=-\frac{\partial \rho V^{2}}{\partial x} .
\end{gathered}
$$

For flow entering the node on the right boundary of the subdomain before the traffic light these conditions in the difference form with the first order of approximation are as follows:

$$
\begin{gathered}
\rho_{N}^{n+1}=\rho_{N}^{n}+\frac{\Delta t}{h}(\rho V)_{N-1}^{n}, \\
V_{N}^{n+1}=0 .
\end{gathered}
$$

For flow exiting the node on the left boundary of the subdomain after the traffic light:

$$
\begin{gathered}
\rho_{0}^{n+1}=\rho_{0}^{n}-\frac{\Delta t}{h}(\rho V)_{1}^{n}, \\
(\rho V)_{0}^{n+1}=(\rho V)_{0}^{n}-\frac{\Delta t}{h}\left(\rho V^{2}\right)_{1}^{n} .
\end{gathered}
$$

Here superscripts correspond to the time levels and subscripts correspond to the points of computational subdomains.

Figure 7 reflects the obtained results for T-type signalized intersection at different traffic light phases. The density values are presented. Black arrows show the direction of cars' movement. The traffic light phases are depicted by the traffic light colours near the roads. The picture at Time 1 demonstrates the initial statement of the problem. At Time 29 one can see the density increase before the red traffic lights. At Times 44 and 90 when the green traffic 
light is on, the density jump in the corresponding section of the road in front of the traffic light begins to decrease.
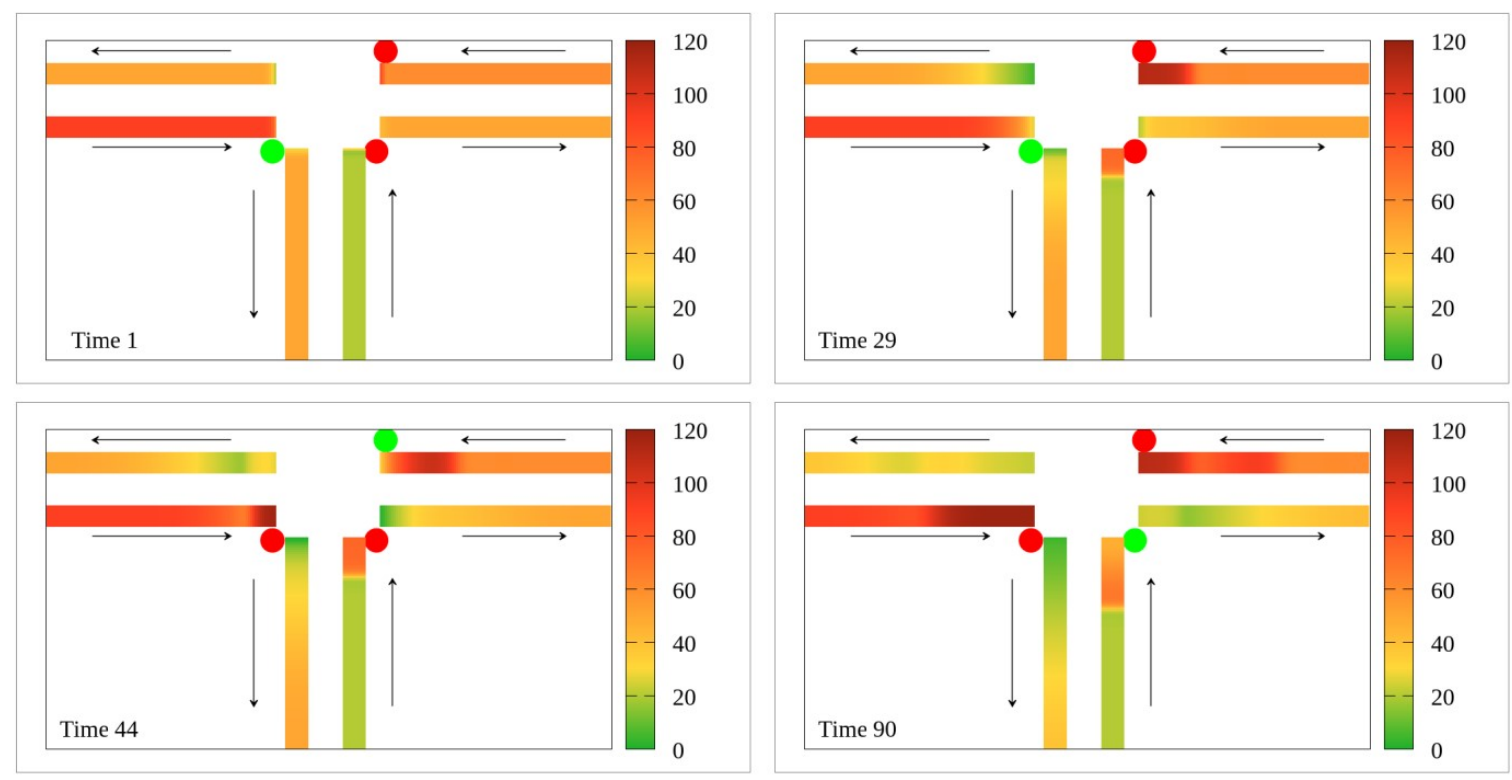

Figure 7. Simulating traffic on T-type signalized intersection at different traffic light phases
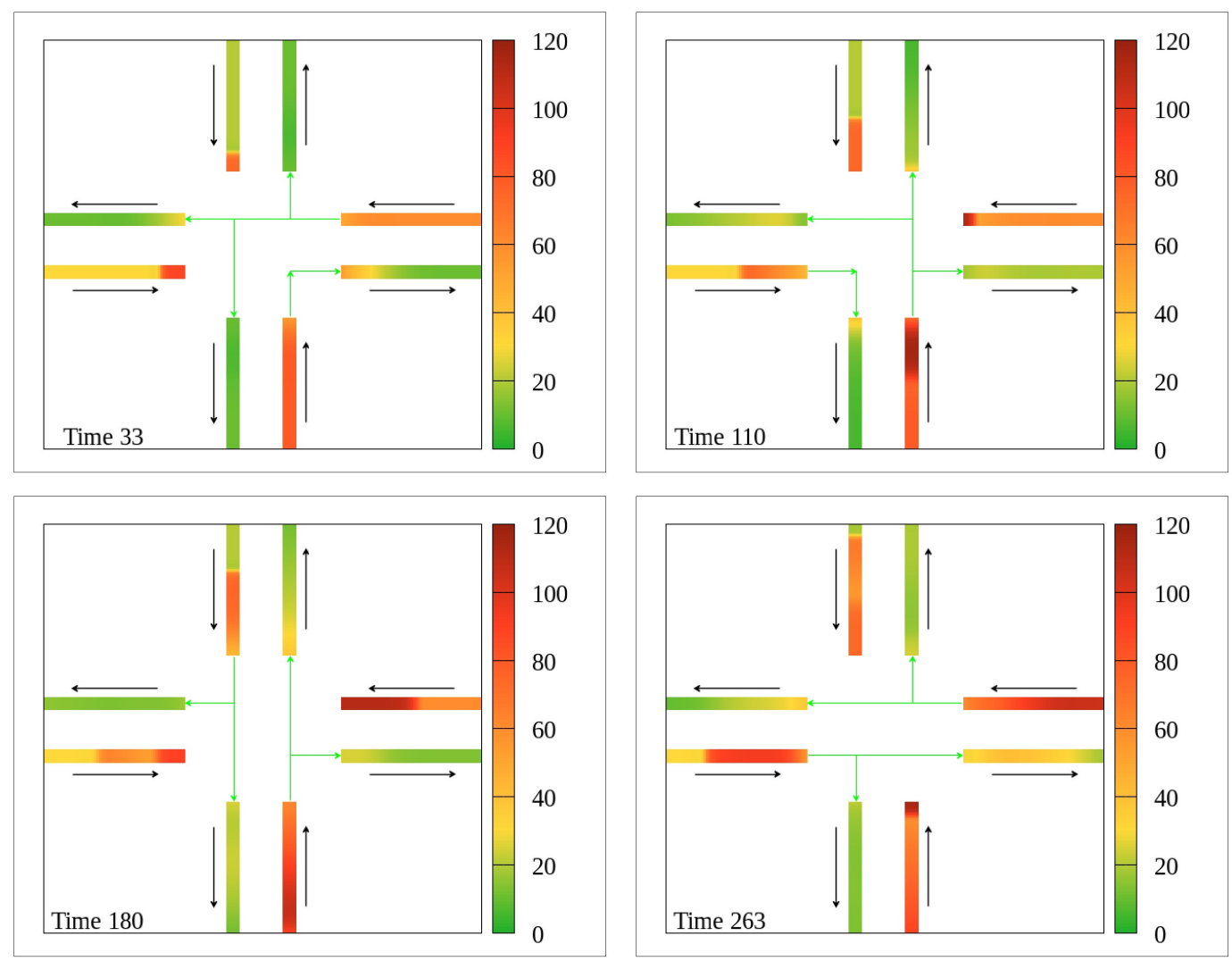

Figure 8. Simulating traffic on X-type signalized intersection at different traffic light phases 
Figure 8 demonstrates the results for X-type signalized intersection. The traffic light phases are depicted by green arrows showing the allowed directions of movement at the intersection during the given phase. In the pictures one can observe the same dependence of the density on traffic signals as in the previous case.

One more test prediction is the simulation of traffic flow on the road with a preset geometry. It should be noted that the simplest test calculations of traffic on the road with an increase and decrease in the number of lanes, as well as a comparison of the results with the calculations from [27] are given in [41]. The following problem statement is considered in this article. The $20 \mathrm{~km}$ road section with the number of lanes $I$ is under consideration. The computational domain is depicted in Fig. 9.

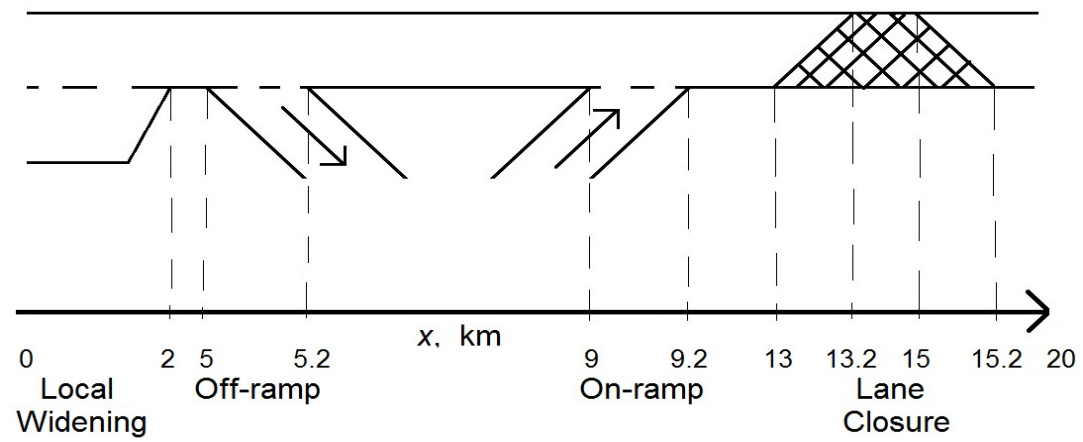

Figure 9. Geometry of the road section under consideration

This section contains:

- a local road widening of $2 \mathrm{~km}$ long,

- an exit (the off-ramp with length $L_{r m p \_o u t}=0.02 \mathrm{~km}$ starting at the point $x=5 \mathrm{~km}$ ),

- $\quad$ an entry (the on-ramp with length $L_{r m p}$ in $=0.02 \mathrm{~km}$ starting at $x=9 \mathrm{~km}$ ),

- a lane closure starting at $x=13.2 \mathrm{~km}$ and lasting $1.8 \mathrm{~km}$.

The volume of traffic (input flows of the entry, exit and main road) is constant and is set by values $Q_{r m p \_i n}, Q_{r m p \_o u t}$ and $Q$.

Results of calculations (the spatiotemporal pattern of the density) are presented in Fig. 10.

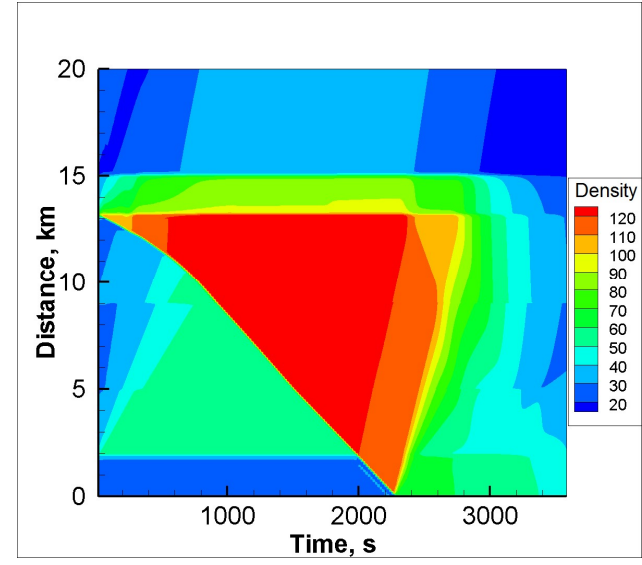

Figure 10. Density field on the road section under consideration 
Obtained results show that significant degradation of the road situation occurs at the lane closure point. The bottleneck capacity can no longer keep up with the input flow and the traffic breakdown takes place at this point, the density jam propagates upstream.

One can conclude that the numerical simulation with the use of the proposed QGD traffic model and the developed parallel software leads to correct results. In the calculations, parallelization efficiency of almost 100 percent was achieved.

Models created can be implemented as a part of Intelligent Transportation System (ITS) of a city, serving the purpose of informing drivers, rerouting if necessary, managing road infrastructure in order to use a transportation network safely and efficiently.

\section{CONCLUSIONS}

The following results obtained should be noted.

The microscopic CA model of traffic flows has been extended by improving the existing algorithms and adding new ones:

- lane changing;

- crossroad overcoming for different types of crossroads;

- forming the entrance queue;

- moving on the road with narrowing/widening;

- driving around an obstacle;

- implementing different driving strategies.

A new version of the CA model, namely the 'slow-to-start' model, has been proposed in order to reproduce the transition from the free flow phase to the synchronized flow phase more realistically.

The program package consisting of two modules - the Computational module and the User Interface and Visualization module - has been created and adapted for supercomputer calculations.

Several test problems have been solved to verify the developed models and algorithms. Vehicle movement on different road fragments, such as a T-cross, an X-cross, an U-turn, an on-ramp, has been predicted, the expected behaviour of movement has been observed. It was shown that traffic on more complex road networks can be simulated by various combinations of the listed road elements.

The one-dimensional variant of the QGD system of equations for the description of vehicular traffic flow on roads with $\mathrm{T}$-cross and $\mathrm{X}$-cross has been proposed. The model can take into account the changing number of lanes, on-ramps and off-ramps. The corresponding test predictions have demonstrated the adequacy of the proposed model. The parallel algorithm based on the principle of geometrical parallelism has been developed. Due to the implementation of the model by explicit difference schemes very high efficiency of parallelization was achieved. The approach can be successfully employed if the average quantities such as the density and the velocity of traffic flow are under consideration. 


\section{REFERENCES}

[1] V.I. Mazhukin, A.V. Shapranov, A.V. Mazhukin, O.N. Koroleva. "Mathematical formulation of a kinetic version of Stefan problem for heterogeneous melting/crystallization of metals". Mathematica Montisnigri, 36, 58-77 (2016).

[2] A.A. Samokhin, V.I. Mazhukin, A.V. Shapranov, M.M. Demin, A.E. Zubko, "Molecular dynamics modeling of nanosecond laser ablation: transcritical regime", Mathematica Montisnigri, 38, 78-88 (2017).

[3] A.A. Samokhin, V.I. Mazhukin, M.M. Demin, A.V. Shapranov, A.E. Zubko, "Molecular dynamics simulation of al explosive boiling and transcritical regimes in nanosecond laser ablation", Mathematica Montisnigri, 41, 55-72 (2018)

[4] T. Kudryashova, Yu. Karamzin, V. Podryga, S. Polyakov, "Two-Scale Computation of N2-H2 Jet Flow Based on QGD and MMD on Heterogeneous Multi-Core Hardware", Advances in Engineering Software, 120, 79-87 (2018)

[5] V. Podryga, S. Polyakov, "Correction of the gas flow parameters by molecular dynamics", Proceedings of IV Int. Conf. on Particle-Based Methods - Fundamentals and Application (PARTICLES 2015), Barcelona, Spain, 779-788 (2015).

[6] M. Cremer, J. Ludwig, "A fast simulation model for traffic flow on the basis of Boolean operations", Math. Comp. Simul., 28, 297-303 (1986).

[7] K. Nagel, M. Schreckenberg, "A cellular automaton model for freeway traffic", J. Phys. I France, 2, 2221-2229 (1992).

[8] S. Maerivoet, B. De Moor, "Cellular automata models of road traffic", Physics Reports, 419 (1), $1-64(2005)$.

[9] Th. Chmura, B. Herz, F. Knorr, Th. Pitz, M. Schreckenberg, "A simple stochastic cellular automaton for synchronized traffic flow", Physica A: Statistical Mechanics and its Applications, 405 (1), 332-337 (2014).

[10] A.P. Buslaev, A.G. Tatashev M.V. Yashina, "On cellular automata, traffic and dynamical systems in graphs", Int. J. of Eng. \& Techn., 7 (2.28), 351-356 (2018).

[11] B. Kerner, S. Klenov, M. Schreckenberg, "Simple cellular automaton model for traffic breakdown, highway capacity, and synchronized flow", Phys. Rev. E, 84, 046110 (2011).

[12] B. Kerner, S. Klenov, G. Hermanns, M. Schreckenberg, "Effect of driver over-acceleration on traffic breakdown in three-phase cellular automaton traffic flow models", Physica A: Statistical Mechanics and its Applications, 392 (18), 4083-4105 (2013).

[13] B. Kerner, The Physics of Traffic, Springer, Berlin, (2004).

[14] H. Yang, J. Lu, X. Hu, J. Jiang, "A cellular automaton model based on empirical observations of a driver's oscillation behavior reproducing the findings from Kerner's three-phase traffic theory", Physica A: Statistical Mechanics and its Applications, 392, 4009-4018 (2013).

[15] H. Jiang, Zh. Zhang, Q. Huang, P. Xie, "Research of vehicle flow based on cellular automaton in different safety parameters", Safety Science, 82, 182-189 (2016).

[16] X. Li, J.-Q. Sun, "Effects of turning and through lane sharing on traffic performance at intersections", Physica A: Statistical Mechanics and its Applications, 444, 622-640 (2016).

[17] K. Gao, R. Jiang, B.-H. Wang, Q.-S. Wu, "Discontinuous transition from free flow to synchronized flow induced by short-range interaction between vehicles in a three-phase traffic flow model", Physica A: Statistical Mechanics and its Applications, 388, (15-16), pp. 32333243 (2009).

[18] M.E. Lárraga, L. Alvarez-Icaza, "Cellular automaton model for traffic flow based on safe driving policies and human reactions", Physica A: Statistical Mechanics and its Applications, 389, (23), 5425-5438 (2010). 
[19] J.S.L. Combinido, M.T. Lim, "Modeling U-turn traffic flow", Physica A: Statistical Mechanics and its Applications, 389 (17), 3640-3647 (2010).

[20] H.B. Zhu, "Numerical study of urban traffic flow with dedicated bus lane and intermittent bus lane", Physica A: Statistical Mechanics and its Applications, 389 (16), 3134-3139 (2010).

[21] J. Vasic, J. Heather, H.J. Ruskin, "Cellular automata simulation of traffic including cars and bicycles", Physica A: Statistical Mechanics and its Applications, 391 (8), 2720-2729 (2012).

[22] S.V. Polyakov, "Exponential finite-difference schemes for diffusion-convection equation", Mathematica Montisnigri, 26, 29-44 (2013).

[23] A. Galanina, A. Favorskiy, "Local two-dimensional perturbations evolution in smallconductivity gas flow in magnetic field", Mathematica Montisnigri, 27, 53-63 (2013).

[24] A.G. Churbanov, O. Iliev, V.F. Strizhov, P.N. Vabishchevich, "Numerical simulation of oxidation processes in a cross-flow around tube bundles", Applied Mathematical Modelling, 59, 251-271 (2018).

[25] A.E. Bondarev, "On the construction of the generalized numerical experiment in fluid dynamics", Mathematica Montisnigri, 42, 52-64 (2018).

[26] M.A. Trapeznikova, N.G. Churbanova, A.A. Lyupa, "Simulation of three-phase fluid flow in a porous medium with account of thermal effects", Mathematica Montisnigri, 33, 105-115 (2015).

[27] M. Treiber, A. Kesting, Traffic Flow Dynamics. Data, Models and Simulation, Springer, BerlinHeidelberg, (2013).

[28] H. Ge, R.J. Cheng, L. Lei, "The theoretical analysis of the lattice hydrodynamic models for traffic flow theory", Physica A: Statistical Mechanics and its Applications, 389 (14), 2825-2834 (2010).

[29] G. Zhang, D. Sun, W. Liu, M. Zhao, S. Cheng, "Analysis of two-lane lattice hydrodynamic model with consideration of drivers' characteristics", Physica A: Statistical Mechanics and its Applications, 422, 16-24 (2015).

[30] G. Peng, Ch. Liu, M. Tuo, "Influence of the traffic interruption probability on traffic stability in lattice model for two-lane freeway", Physica A: Statistical Mechanics and its Applications, 436, 952- 959 (2015).

[31] J.-L. Cao, Zh.-K. Shi, "Analysis of a novel two-lane lattice model on a gradient road with the consideration of relative current", Communications in Nonlinear Science and Numerical Simulation, 33, 1-18 (2016).

[32] J. Zhou, H.L. Zhang, C.P. Wang, Z.K. Shi, "A new lattice model for single-lane traffic flow with the consideration of driver's memory during a period of time", Int. J. of Modern Physics C, 28 (7), 1750086 (2017).

[33] D. Jin, J. Zhou., H.L. Zhang, C.P. Wang, Z.K. Shi, "Lattice hydrodynamic model for traffic flow on curved road with passing", Nonlinear Dynamics, 89 (1) 107-124 (2017).

[34] R. Kaur, S. Sharma, "Analysis of driver's characteristics on a curved road in lattice model", Physica A: Statistical Mechanics and its Applications, 471, 59-67 (2017).

[35] A.B. Sukhinova, M.A. Trapeznikova, B.N. Chetverushkin, N.G. Churbanova, "Twodimensional macroscopic model of traffic flows", Mathematical Models and Computer Simulation, 1 (6), 669-676 (2009).

[36] B.N. Chetverushkin, Kinetic Schemes and Quasi-Gas Dynamic System of Equations, CIMNE, Barcelona, (2008).

[37] A. Chechina, N. Churbanova, M. Trapeznikova, "Reproduction of experimental spatio-temporal structures in traffic flows using mathematical model based on cellular automata theory", Periodicals of Engineering and Natural Sciences, 7 (1), 76-81 (2019).

[38] A. Chechina, N. Churbanova, M. Trapeznikova, A. Ermakov, M. German, "Traffic flow modeling on road networks using cellular automata theory", Int. J. of Engineering \& Technology, 7 (2.28) 225-227 (2018). 
[39] A. Chechina, N. Churbanova, M. Trapeznikova, "Multilane traffic flow modeling using cellular automata theory", EPJ Web of Conferences, 173, 06003 (2018).

[40] A.A. Samarskii, The Theory of Difference Schemes, CRC Press, (2001).

[41] P.A. Sokolov, I.V. Shkolina, M.A. Trapeznikova, A.A. Chechina, N.G. Churbanova, "Modelirovanie dvizheniya avtotransporta na osnove KGD sistemy` uravnenij s ispol`zovaniem superkomp 'yuterov", T-Comm, 13 (6), 46-52 (2019).

[42] KIAM - The official site of Keldysh Institute of Applied Mathematics, "Hybrid computational cluster MVS-Express" available at: http://www.kiam.ru/MVS/resourses/mvse.html. (Accessed November 19, 2019)

[43] Aimsun Transport Simulation Systems https://www.aimsun.com/ (Accessed November 19, 2019)

Received October 14, 2019 